\title{
Arbeitsbeziehungen in der Zerreißprobe? Reformen in Italien und Spanien während der Großen Rezession
}

Trotz der breiten Debatte über den Globalisierungsprozess bleibt der Einfluss internationaler Organisationen auf die Arbeitsbeziehungen in der wissenschaftlichen Literatur weitgehend unbeachtet: Die Analyse industrieller Beziehungen konzentrierte sich lange Zeit auf nationale Institutionen, während sich die Literatur zum Arbeitsprozess auf die Unternehmensebene oder auf das kapitalistische System im Allgemeinen bezieht. Angesichts der drastischen Reformen in Ländern Südeuropas, die seit 2010 unter direktem Druck der Europäischen Kommission, der Europäischen Zentralbank (EZB) und des Internationalen Währungsfonds (IWF) sowie unter indirektem Einfluss der deutschen Regierung verabschiedet wurden, drängen sich interessante Fragen zu den Wechselbeziehungen zwischen staatlichen und überstaatlichen Akteuren auf. ${ }^{1}$

GUGLIELMO MEARDI

\section{Einleitung}

Der vorliegende Beitrag untersucht die Reformen der Arbeitsbeziehungen in Spanien und Italien während der Großen Rezession. Bislang mussten diese beiden Länder weder Rettungspakete des IWF zur Abwendung eines Staatsbankrotts in Anspruch nehmen, noch waren sie direkter Aufsicht durch diesen unterstellt (wenngleich Spanien im Juni 2012 bei der Bankenrettung Hilfe benötigte). Dennoch sahen sich beide Staaten durch die Staatsschuldenkrise zur Erfüllung beispielloser, von der EU-Kommission und der EZB diktierter Auflagen genötigt. Im Folgenden werden die Reformpfade der beiden Länder vor dem Hintergrund der krisenbedingten Veränderungen des politischen Instrumentariums der Europäischen Union (EU) und mit Bezug auf den aktuellen Diskussionsstand des sogenannten mediterranen Kapitalismus- und Arbeitsbeziehungsmodells rekonstruiert. Die Analyse stützt sich auf Primär- und Sekundärquellen und deutet auf einen institutionellen Umbruch hin, der auf Verdrängung (displacement) lokaler durch fremde Muster beruht und schneller abläuft als in Theorien institutionellen Wandels gemeinhin angenommen (Streeck/Thelen 2005). Der Prozess wird eher von ausländischen als von innerstaatlichen Akteuren vorangetrieben und fördert das Entstehen „entbetteter” oder „unzusammenhängender“ (disconnected) Spielarten des Kapitalismus (Thompson 2003), in denen soziale Aspekte des Beschäftigungsverhältnisses (wie Loyalität,
Engagement, Status oder Mitspracherechte) keine hinreichende Berücksichtigung finden. Die entstehenden Ordnungen zeichnen sich durch einen Mangel an Stabilität aus, weshalb Gegenreaktionen zu ihrer Stabilisierung zu erwarten sein dürften - ob nun in eine konservative oder eine progressive Richtung weisend.

\section{Internationale Organisationen und Umbrüche im Bereich der Arbeitsbeziehungen}

Ungeachtet der wachsenden Debatte über den Globalisierungsprozess galten Arbeitsbeziehungen und Arbeitsprozess in der Wissenschaft lange Zeit als ein weitgehend geschlossenes System aus Arbeitgebern und Arbeitnehmern mit eventueller Beteiligung von Regierungsbehörden oder anderen lokalen Institutionen. Insbesondere die institutionelle Betrachtungsweise hielt an der Vorstellung fest, dass sich die industriellen Beziehungen unter globalem und internationalem Druck pfadabhängig entwickeln (siehe zum Beispiel

1 Übersetzung aus dem Englischen: Lisa Eskuche. 
Traxler et al. 2001). Aus Sicht des Varieties of CapitalismAnsatzes fördert der wirtschaftliche Internationalisierungsprozess infolge der strategischen Ausnutzung institutioneller Unterschiede durch internationale Investoren die nationale Spezialisierung und sorgt dadurch für eine verstärkte Auseinanderentwicklung der Länder (Hall/Soskice 2001). Diese Auffassung hat sich trotz mancher Einwände gegen die angenommene Einheitlichkeit und Kontinuität nationaler Kapitalismusmodelle, insbesondere im Zusammenhang mit der Globalisierung der Finanzmärkte, auf dem Forschungsgebiet der industriellen Beziehungen als vorherrschende Meinung durchgesetzt (Streeck 2009).

Bislang galt das Interesse an der Rolle überstaatlicher Akteure in erster Linie peripheren und wirtschaftlich abhängigen Ländern wie den postkommunistischen Staaten (siehe beispielsweise Upchurch/Weltman 2008) und nur ausnahmsweise dem kapitalistischen Westen. Augenfälligstes Beispiel hierfür ist die weit verbreitete Einschätzung, die EU-Politik sei im sozialen Bereich reine Kosmetik (Streeck 1995). Seinem institutionalistischen Ansatz getreu wendete sich Colin Crouch Anfang der 1990er Jahre gegen die Annahme, die Europäische Wirtschaftsgemeinschaft könne im Bereich der industriellen Beziehungen internationale Konvergenz herbeiführen: „to date there is little sign that systematic differences of approach to the occupancy of political space are even perceived by policy-makers, let alone have become an object of harmonization" (Crouch 1993, S. 350). Mit der 2007 einsetzenden Wirtschaftskrise rückte die Frage nach der Rolle internationaler Wirtschaftseinflüsse verstärkt in den Fokus. Es wurde argumentiert, Krisen und Wirtschaftsschocks böten internationalen Akteuren Gelegenheit, Volkswirtschaften umzustrukturieren (Stiglitz 2002; Klein 2008). Die Finanzkrise hat dem neoliberalen Wirtschaftskonzept wohl nicht den Boden entzogen, aber sicher eine Anpassung erzwungen (Crouch 2011).

Die Gründe dafür liegen zumindest teilweise in der veränderten Rolle der EU. Während herkömmliche EU-Politik in Form von "harten“ und „weichen“ Maßnahmen (Richtlinien bzw. Koordination) die Beschäftigungslage bislang wenig beeinflusst hatte, löste die Staatsschuldenkrise verstärkte Eingriffe supranationaler Institutionen in die industriellen Beziehungen derjenigen Länder aus, die Rettungspakete oder Interventionen der EZB auf dem Staatsanleihenmarkt in Anspruch nehmen mussten. Die Auswirkungen sind vor allem in Südeuropa zu erkennen. Innerhalb von wenigen Monaten wurden in verschiedenen Ländern der Region ohne lange Diskussionen oder gesellschaftliche Verhandlungsprozesse Reformen verabschiedet, die in ihrer einschneidenden Wirkung alle Eingriffe der letzten 20 Jahre oder mehr in den Schatten stellen. Sollte die Durchsetzung dieser auf Dezentralisierung der Tarifverhandlungen, Lockerung des Kündigungsschutzes und Anhebung des Rentenalters abzielenden Reformmaßnahmen in der Praxis gelingen, wären grundlegende Veränderungen des sogenannten mediterranen Beschäftigungs- und Sozialmodells regulierter Güter- und Arbeitsmärkte die Folge (Amable 2003).
Im Zuge der Staatsschuldenkrise wurden in der EU und speziell in der Eurozone neue, wirksamere wirtschaftspolitische Steuerungsinstrumente eingeführt: ein „Europäisches Semester" der Überprüfung nationaler Haushalte, ein auch als „Six-Pack“ betiteltes sechsteiliges Gesetzespaket zur Vermeidung makroökonomischer Ungleichgewichte, in dem unter anderem die Lohnstückkosten Erwähnung finden, sowie ein Europäischer Fiskalpakt, mit dem größere Haushaltsdisziplin erzwungen werden soll (Erne 2012). Sowohl die Strukturreformen als auch der Six-Pack bedeuten in dreifacher Hinsicht eine Abkehr von den bisherigen Gepflogenheiten der EU: erstens, von den EU-Verträgen, welche Lohnbildung und Tarifwesen ausdrücklich von der Zuständigkeit der Gemeinschaftspolitik ausnehmen (Artikel 153 des Vertrags von Lissabon); zweitens, vom Leitbild eines sozialen Europas mit einem Sockel an Grundrechten - die aktuelle Reformwelle stellt das Prinzip wirtschaftlichen Wettbewerbs implizit über soziale Ansprüche - sowie, drittens, die Abkehr von „weicher“ Arbeitsmarktregulierung und der Übergang zu einer ausgesprochen harten Politik: Ein neu eingeführtes, striktes Verfahren „multilateraler Überwachung“" ahndet Verstöße mit Geldstrafen, und die empfohlenen Strukturreformen, wenn diese auch nicht rechtsverbindlich sind, lassen in der Praxis keine andere Alternative zu als den Staatsbankrott.

Im Rahmen der Wachstumsstrategie „Europa 2020“ spielen aus Sicht der Europäischen Kommission und der EZB zwei Maßnahmenvorschläge seit 2010 eine besondere Rolle. Erstens wurde sowohl in Spanien als auch in Italien die Durchsetzung neuer Rahmenbedingungen für Lohnverhandlungen angeregt, mit denen die Lohnentwicklung an das Produktivitätswachstum auf Branchen- bzw. Unternehmensebene gekoppelt werden soll (Europäische Kommission 2012) kurzum, es wird einer Dezentralisierung des Tarifwesens der Weg bereitet. Zweitens geht es um die Lockerung des Kündigungsschutzes: Der Vorschlag eines flexiblen, „einfachen, unbefristeten Arbeitsvertrags" im Rahmen der Agenda für neue Kompetenzen und neue Beschäftigungsmöglichkeiten (Europäische Kommission 2010) zielt darauf ab, die Segmentierung des Arbeitsmarktes zu überwinden. Es deutet jedoch wenig darauf hin, dass diese oder ähnlich gelagerte Vorschläge positive Effekte für Wirtschaft oder Arbeitsmarkt zeitigen (Esping-Andersen/Regini 2000; Traxler et al. 2001). Überdies stehen sie im Widerspruch zu den im Jahre 2006 von der vorherigen EU-Kommission in ihrem Bericht Beschäftigung in Europa (Europäische Kommission 2006) gemachten Äußerungen, in denen sie die Bedeutung eines gesetzlichen Kündigungsschutzes für die Investition in Humankapital hervorgehoben und koordinierte Tarifverhandlungen mit stabilen Lohnentwicklungen und Produktivitätssteigerungen in Verbindung gebracht hat.

Zur gleichen Zeit, als sich in der Europäischen Kommission und im Europäischen Rat die Macht von den Abteilungen für Beschäftigung und Soziales zu den Finanzabteilungen verschob und ein vermehrter Einfluss der EZB spürbar wurde, verloren die Institutionen das Interes- 
se an wissenschaftlichem Fachwissen über die sozialen Aspekte des Arbeitsmarktes und verlegten sich auf wenig differenzierte Flexibilitäts- und Dezentralisierungsziele.

Bemerkenswert an dieser Entwicklung ist, dass die Reforminhalte von supranationalen Institutionen diktiert wurden und nicht unbedingt den Forderungen der Arbeitgeber auf nationaler Ebene entsprechen. Wenngleich sich die umgesetzten Reformen als arbeitgeberfreundlich bezeichnen lassen, standen die Ziele einer radikalen Dezentralisierung des Tarifwesens und der Aufweichung des Kündigungsschutzes nicht auf der Wunschliste der lokalen Arbeitgeberverbände. Diese sprechen sich vielmehr für einen zumindest teilweise koordinierten Lohnfindungsprozess aus (um Lohnverhandlungen auf Unternehmensebene und die damit verbundenen Transaktionskosten zu vermeiden) und betrachten die Spaltung des Arbeitsmarkts in Kernbeschäftigte und „periphere“ Arbeitnehmer als unverzichtbares Mittel der Personalsteuerung. Auch die Gewerkschaften sahen sich durch den äußeren Druck ausmanövriert. Nachdem traditionelle Kampfmethoden versagt hatten, bemühten sich die Arbeitnehmerorganisationen auf nationaler Ebene um politische Kompromisse, mussten aber feststellen, dass ihre Vereinbarungen zunehmend auf tönernen Füßen standen.

\section{Arbeitsbeziehungen im mediterranen Raum und EU-Reformen}

Trotz eines ähnlichen Rückgangs des Bruttoinlandsproduktes (BIP) im Jahr 2009 (-3,7\% in Spanien und $-5.2 \%$ in Italien) ist die Arbeitsmarktentwicklung in den beiden Ländern recht unterschiedlich verlaufen. Spanien verzeichnete im Vergleich zur übrigen EU einen besonders dramatischen Anstieg der Arbeitslosenzahlen (2007-2009 von 7 auf 20\%). Italien dagegen verlegte sich auf das Horten von Arbeitskräften und führte zur Vermeidung von Stellenkürzungen Kurzarbeit ein (der Anteil der Arbeitslosen in Italien stieg im genannten Zeitraum von 6 auf 7,5\%). 2010 und besonders 2011 waren beide Länder von der Staatsschuldenkrise betroffen, allerdings aus unterschiedlichen Gründen. Italien wies ein geringes Haushaltsdefizit und sogar einen Primärüberschuss auf, trug aber gleichzeitig schwer an einer hohen kumulierten Schuldenlast (2010: $118 \%$ des BIP). Umgekehrt war in Spanien die öffentliche Verschuldung relativ niedrig (2010: 61 \% des BIP), während das Haushaltsdefizit wuchs (2009: 11,2\%). In beiden Ländern führten die finanziellen Engpässe $2011 \mathrm{zu}$ einem Regierungswechsel und zu intensivem Druck der europäischen Institutionen, die auf Reformen der öffentlichen Finanzen und der Arbeitsmarktpolitik drängten. Die folgende Analyse länderspezifischer Entwicklungen basiert auf 18 im Zeitraum 2011-2012 geführten Leitfadeninterviews mit führenden Experten der wichtigsten Gewerkschaften, Arbeitgeberverbände und Regierungsparteien in beiden Ländern sowie auf EU-Ebene. Darüber hin- aus wurden alle relevanten Dokumente (Texte von Reformgesetzen, Vereinbarungen sowie von Resolutionen tripartistischer Gremien) analysiert und die einschlägige öffentliche Diskussion der Sachverhalte in den Jahren 2011 und 2012 durch eine systematische Auswertung der führenden landesweiten Tageszeitungen La repubblica und El País für diesen Zeitraum rekonstruiert.

\subsection{Der Fall Italien}

Dem Kommissionsbericht Beschäftigung in Europa 2006 (Europäische Kommission 2006) zufolge ist Italien heute nicht mehr der traditionellen Gruppe mediterraner Ökonomien mit geringer Arbeitsmarktflexibilität und geringer bis mittlerer Beschäftigungssicherheit zuzurechnen, sondern der von hoher Flexibilität und geringer Sicherheit geprägten osteuropäischen Gruppe, deren Arbeitsmarktpolitik in der Literatur auch mit dem Begriff „flex-insecurity“ beschrieben wird (Berton et al. 2012).

Dieser Wechsel ist weitgehend den 1997 und 2003 verabschiedeten Arbeitsmarktreformen zu verdanken, die nach Verhandlungen mit Arbeitgeberverbänden und einigen beziehungsweise allen Gewerkschaften Gesetzeskraft erlangten. Dabei war es gelungen, radikale Reformmaßnahmen abzuwenden: Mit einer 3 Mio. Teilnehmer starken Demonstration in Rom und einem Generalstreik in den Jahren 2002 beziehungsweise 2003 zwang die mitgliederstärkste Gewerkschaft Italiens, CGIL (Confederazione Generale Italiana del Lavoro), die Regierung zur Rücknahme der angekündigten Aufweichung des Kündigungsschutzes (Reform von Artikel 18 des Statuto dei Lavoratori von 1970).

Nach Anbruch der Krise konnte Italien anfänglich einen Anstieg der Arbeitslosenzahlen vermeiden, hauptsächlich dank staatlich subventionierter Arbeitszeitverkürzung (Lohngarantiefonds Cassa Integrazione und contratti di solidarietà [Kurzarbeit]). Tarifautonomie und Gewerkschaften bewiesen in Italien mehr Widerstandskraft als in den meisten europäischen Ländern; seit den 1980er Jahren hatten sich weder bei der tariflichen Deckungsrate noch beim gewerkschaftlichen Organisationsgrad wesentliche Veränderungen ergeben. Die erhoffte konjunkturelle Erholung blieb jedoch aus, gleichzeitig war Italien aufgrund der in den 1980er Jahren angehäuften öffentlichen Schulden äußerst verwundbar.

Im Januar 2009 einigten sich die Arbeitgeber und die Gewerkschaftsbünde CISL (Confederazione Italiana Sindacati Lavoratori) und UIL (Unione Italiana del Lavoro) auf eine Begrenzung der Lohnindexierung. Die stärkste Gewerkschaft, CGIL, weigerte sich, die Reformvereinbarung zu unterzeichnen, und schränkte dadurch deren Anwendbarkeit ein.

\subsubsection{Der Ausstieg von Fiat aus dem italieni- schen Tarifsystem}

2010 - 2011 beschloss Italiens größter Industriekonzern Fiat (zu dem auch die Firma Chrysler gehört) unter dem Vor- 
standsvorsitzenden Sergio Marchionne, weit über die oben beschriebene Reform hinausgehende Änderungen. Marchionne drohte mit einer Produktionsverlagerung nach Polen und erzwang so die Zustimmung von CISL und UIL sowie von einer Mehrheit der Belegschaft zum Ausstieg aus dem Branchentarifvertrag der Metallindustrie und zum Abschluss betrieblicher Vereinbarungen. Letztere erlangten Ende 2011 Gültigkeit für den gesamten Fiat-Konzern und führten neben einer verstärkten Flexibilisierung der Arbeitszeit auch eine Friedenspflicht der Arbeitnehmer ein.

Die Betriebsvereinbarungen des Fiat-Konzerns waren von historischer Bedeutung. Seit 1984 hatte Italien mit der Illusion eines Branchentarifsystems mit erga-omnes-Wirkung $^{2}$ gelebt. Obgleich Branchentarifverträge streng gesehen nicht rechtsverbindlich sind, zogen die Gerichte bei Streitigkeiten über die Höhe des von der Verfassung garantierten Anspruchs auf gerechte Entlohnung und faire Arbeitszeiten routinemäßig Branchenvereinbarungen als Maßstab heran. Das hatte zur Folge, dass sich praktisch alle Arbeitgeber an Letztere hielten. Mit dem Ausstieg aus den Verhandlungen auf Verbandsebene zog Fiat sich gleichzeitig auch aus der 1993er Vereinbarung zur Vertretung der Gewerkschaften auf Betriebsebene zurück, mit der ein Hybridsystem betrieblicher Interessenvertretung, die rappresentanze sindacali unitarie, entstanden war. Fiat war infolgedessen nur noch an das Statuto dei Lavoratori (Gesetz Nr. 300 von 1970) gebunden, das nur diejenigen Gewerkschaften schützt, die Tarifverträge unterzeichnet haben. Die Metallgewerkschaft FIOM (Federazione Italiana Operai Metallurgici, eine Mitgliedsgewerkschaft des CGIL) weigerte sich, die neue FiatBetriebsvereinbarung zu unterzeichnen, und fand sich in der Konsequenz von den Betriebsstätten des Konzerns ausgeschlossen - trotz ihres Status als weitaus mitgliederstärkste Gewerkschaft der italienischen Fiat-Werke und der gesamten Metallindustrie. Durch den Ausstieg aus dem italienischen Tarifsystem legte sich Fiat zugleich mit dem Arbeitgeberverband Confindustria an, der zwar einerseits mehr Flexibilität fordert, andererseits aber Branchenvereinbarungen bevorzugt.

Am 28. Juni 2011 wurde ein neues tripartistisches Abkommen zur Reform der Branchentarifabschlüsse unterzeichnet - diesmal auch vom Gewerkschaftsbund CGIL, während die FIOM sich weiterhin sperrte. Die Vereinbarung lässt sich im Endergebnis als eine Art von organisierter Dezentralisierung beschreiben. Sie sicherte den Fortbestand der Branchenabschlüsse und entsprach damit den Interessen der kleinen und mittelständischen Unternehmen (KMU), die angesichts der entsprechenden Transaktionskosten (sowie aufgrund der paternalistischen Mentalität der meisten kleinen und mittelständischen Arbeitgeber Italiens) Tarifverhandlungen auf Unternehmensebene zu vermeiden suchen. Für Fiat ging die Vereinbarung jedoch aufgrund mangelnder Rückwirkung nicht weit genug. Am 18. Juli wurde die erste Betriebsvereinbarung für ein Werk des Fiat-Konzerns (Werk Pomigliano) vor Gericht für rechtens befunden, der damit verbundene Ausschluss der FIOM jedoch nicht.
Fiat forderte zur Absicherung seiner Rechtsposition die Schaffung einer gesetzlichen Grundlage. Von den in der Verfassung von 1948 festgeschriebenen allgemeinen Grundsätzen abgesehen, war das italienische Tarifwesen jedoch nie einer gesetzlichen Regelung unterworfen gewesen. ${ }^{3}$

\subsubsection{Intervention der EZB und die politischen Reaktionen}

Im August 2011 spitzte sich die Situation angesichts der finanziellen Turbulenzen in der Eurozone zu. Der Spread für zehnjährige italienische Staatsanleihen kletterte gegenüber den deutschen Referenzanleihen auf über $5 \%$ und näherte sich damit dem Punkt, an dem Griechenland, Irland und Portugal sich gezwungen gesehen hatten, um ein finanzielles Rettungspaket zu ersuchen. Confindustria und die drei führenden Gewerkschaften forderten einen Sechs-Punkte-Plan ein, der - was in diesem Zusammenhang besonders relevant erscheint - vage Anspielungen auf Arbeitsmarkt-Deregulierung und Tarifreform enthielt. Am 5. August forderten der amtierende und der designierte Präsident der EZB, JeanClaude Trichet und Mario Draghi, die italienische und die spanische Regierung in gemeinsam unterzeichneten, separaten Schreiben zu Austeritätsmaßnahmen sowie Strukturund Verfassungsreformen auf. Implizit waren diese als Vorbedingungen für den für die darauffolgende Woche geplanten Ankauf italienischer (und spanischer) Staatsanleihen auf dem Sekundärmarkt gedacht. Die Schreiben wurden zwar nicht offiziell bekannt gemacht, der Inhalt des Briefes an die italienische Regierung jedoch Ende September von der italienischen Tageszeitung Corriere della Sera veröffentlicht (Trichet/Draghi 2011). In dem Schreiben war die Rede von unumgänglichen Maßnahmen („essential measures“) auf dem Arbeitsmarkt, mit dem Zusatz, dass die aufgeführten Reformen baldmöglichst per Dringlichkeitsverordnung umzusetzen und vom Parlament bis Ende September zu bestätigen seien. Die beiden Hauptforderungen betrafen, erstens, zusätzliche Reformen des Tarifsystems mit dem Ziel, durch Vereinbarungen auf Unternehmensebene die Anpassung von Löhnen und Arbeitsbedingungen an die spezifischen Bedürfnisse der Firmen zu ermöglichen und die Relevanz dieser Abschlüsse für andere Verhandlungsebenen zu steigern („to further reform the collective wage bargaining system allowing firm-level agreements to tailor wages and working conditions to firms' specific needs and increasing their relevance with respect to other layers of negotiations") sowie, zweitens, eine umfassende Überprüfung der Vorschriften zur Einstel-

2 Erga omnes (lateinisch "gegenüber allen“) meint hier absolutes Recht. Dies wirkt sich nicht nur auf die Tarifparteien, sondern auf alle bei dem betreffenden Arbeitgeber Beschäftigten aus.

3 Das einzige Tarifgesetz in der Geschichte Italiens, 1961 verabschiedet, wurde als Verstoß gegen die Koalitionsfreiheit gewertet und für verfassungswidrig erklärt. 
lung und Entlassung von Mitarbeitern („a thorough review of the rules regulating the hiring and dismissal of employees") (Trichet/Draghi 2011). Die EZB empfahl zudem, nach dem Vorbild der kurz zuvor erfolgten Verfassungsänderung in Deutschland eine Schuldenbremse in die italienische Verfassung aufzunehmen.

Die italienische Regierung unter Berlusconi reagierte auf dieses (damals noch unveröffentlichte) Schreiben am Samstag, dem 13. August mit der Erstellung eines Sparhaushaltes und dem Entwurf eines Reformgesetzes. Am folgenden Montag begann die EZB mit dem Ankauf italienischer und spanischer Staatsanleihen. Der 15. August ist in Italien ein Feiertag und überhaupt sind in diesem Monat die meisten Arbeitsstätten wegen Betriebsferien geschlossen - folglich konnten keine Protestaktionen der Gewerkschaften erfolgen. Am 18. August kündigte Berlusconi unter Umgehung einer parlamentarischen Debatte Dringlichkeitsmaßnahmen an, die sämtliche Forderungen der EZB erfüllten: einen neuen Sparhaushalt, Arbeitsmarktreformen und den Entwurf eines Gesetzes zur Verfassungsänderung.

Nachdem der Sparhaushalt den Verhandlungsprozess in den Regierungskoalitionen durchlaufen hatte, enthielt die endgültige Fassung nicht mehr die ursprünglich vorgesehene Kürzung der staatlichen Rente, sondern nur noch eine Anhebung des Rentenalters für Frauen. Die Arbeitsmarktreformen dagegen wurden in vollem Umfang beibehalten und gingen deutlich über frühere Reformen hinaus. Dazu gehörte auch die Reform des Tarifwesens, und Fiat zuliebe wurde auch das Rückwirkungsverbot ausdrücklich aufgehoben. Das neue Gesetz eröffnet für betriebliche Vereinbarungen die Möglichkeit, nicht nur von Branchenvereinbarungen abzuweichen, sondern auch gesetzliche Regelungen zum Arbeitnehmerschutz einschließlich der Kündigungsschutzregelungen zu umgehen. Damit war Artikel 18 des seit 1970 unangetasteten und 2002/2003 vom CGIL erfolgreich verteidigten Statuto dei Lavoratori potenziell ausgehöhlt. Die Gewerkschaften reagierten empört und gelobten öffentlich, keine gegen Artikel 18 verstoßenden Unternehmensvereinbarungen zu unterzeichnen. Am 21. September trafen sie mit dem Arbeitgeberverband Confindustria eine neue bilaterale Vereinbarung, in der sich die Parteien verpflichteten, auf Unternehmensebene nur über Sachverhalte zu verhandeln, zu denen sich die Verbände zuvor auf nationaler Ebene geeinigt haben. Im Kern läuft diese Maßnahme auf ein Vetorecht der Gewerkschaften in Verhandlungen hinaus, die Artikel 18 berühren. Fiat zog sich unter Protest aus Confindustria zurück und stürzte den Verband damit in politische und finanzielle Schwierigkeiten, die sich unter anderem in einer Mitgliederflucht äußerten: 2012 sank das Beitragsaufkommen des Verbands um $12 \%$. Das Interesse der Arbeitgeber an der betrieblichen Vereinbarung von Ausnahmeregelungen nach dem neuen Gesetz hielt sich anfänglich stark in Grenzen. Nach einer 2011 von der Banca d'Italia durchgeführten Umfrage scheuten die Unternehmen die mit Verhandlungen verbundenen Transaktionskosten, gewerkschaftliche Protestaktionen sowie die mit dem Übergang zu
Unternehmensvereinbarungen einhergehende allgemeine Ungewissheit.

\subsubsection{Reformen unter Monti}

Die Staatsschuldenkrise war jedoch noch nicht vorüber. Im November 2011 sah sich Italien nicht nur mit weiteren Forderungen der EU-Kommission und der EZB konfrontiert, sondern auch mit einem ausgeprägten Vertrauensschwund der Finanzmärkte. Die Renditezuschläge für italienische Staatsanleihen stiegen auf bis dato unerreichte Höhen. Berlusconi trat zurück und wurde unverzüglich durch den früheren Binnenmarktkommissar Mario Monti ersetzt, der eine technokratische Regierung mit breiter Parlamentsmehrheit bildete. Monti verabschiedete umgehend ein drastisches Sparpaket mit Kürzungen in Höhe von $30 \mathrm{Mrd}$. $€$, das unter anderem die Anhebung des Rentenalters vorsah. Die Gewerkschaften reagierten mit einem Aufruf zu einem dreistündigen Generalstreik am 12. Dezember, der jedoch kaum befolgt wurde und wirkungslos verpuffte.

Am 12. März 2012 kündigte die Regierung Maßnahmen zur Arbeitsmarktreform an, die zum ersten Mal Artikel 18 direkt berührten. Die Sozialpartner wurden zwar angehört, doch fanden im Gegensatz zum Konzertierungsverfahren der 1990er Jahre keine wirklichen Verhandlungen und keine Vereinbarungsunterzeichnung statt. Der CGIL rief einen 16-stündigen Generalstreik aus und erreichte minimale Zugeständnisse. Im Juli 2012 wurden die Reformmaßnahmen vom Parlament verabschiedet.

Die Erklärung von Arbeitsministerin Elsa Fornero, die Maßnahmen seien „nicht von der EU diktiert“ worden, wurden weitgehend als prophylaktische Entschuldigung und Beweis des Gegenteils aufgefasst. Die Arbeitgeberverbände selbst hatten wenig Begeisterung für Reformmaßnahmen gezeigt; ihre Zurückhaltung kam in der Unterzeichnung der bilateralen Vereinbarung vom 21. September deutlich zum Ausdruck. Für Kleinunternehmer spielt Artikel 18 keinerlei Rolle, da seine Bestimmungen erst ab einer Belegschaftsgröße von 15 Mitarbeitern greifen. In unseren Gesprächen betrachteten sowohl die Vertreter von Assolombarda (dem Arbeitgeberverband der Region Mailand und einflussreichsten Confindustria-Mitglied) als auch die italienische American Chamber of Commerce das Thema Artikel 18 als Nebenschauplatz im Vergleich zu Sachverhalten wie der komplexen Vielfalt von Beschäftigungsverträgen und der notorisch langen Dauer arbeitsgerichtlicher Verfahren (bei Kündigungsverfahren bis zu acht Jahren). Assolombarda-Vertreter bestritten, dass Artikel 18 aus Sicht der KMU eine Wachstumsbremse sei, und sahen das wirkliche Problem in der von individuellen Persönlichkeiten und Familienbanden bestimmten Führungskultur, die sich schwer mit größeren organisatorischen Dimensionen und Beziehungen formellerer Art vereinbaren lasse. Am Tage nach der Ankündigung der Reform zog Confindustria bei der Wahl des neuen Verbandspräsidenten den moderaten Squinzi dem von Fiat favorisierten radikaleren Kandidaten 
vor. Auf erhebliche Kritik stießen die Reformmaßnahmen auch bei der katholischen Kirche.

Im Mittelpunkt von Forneros (explizit am dänischen Modell orientiertem) Reformprogramm standen sowohl Flexibilität als auch Sicherheit, wenngleich der vorgeschlagene einheitliche Arbeitsvertrag fallengelassen wurde. Was die Absicherung anbetrifft, wurde eine umfassende neue Arbeitslosenversicherung (ASPI) eingeführt; frühere Regelungen wurden abgeschafft oder eingeschränkt, die größere Unternehmen und damit die Kernklientel der Gewerkschaften begünstigten. Im Bereich der Arbeitsflexibilität ersetzten die neuen gesetzlichen Regelungen im Falle von Kündigungen aus wirtschaftlichen Gründen das Wiedereinstellungsrecht durch eine finanzielle Entschädigung in Höhe von bis zu 27 Monatsgehältern. Unter dem Druck der Gewerkschaften und der politischen Linken wurde zumindest in Ansätzen eine gerichtliche Überprüfung der wirtschaftlichen Begründung eingeführt, um diskriminierenden Praktiken einen Riegel vorzuschieben. Confindustria zeigte sich bedingt zufrieden, meldete aber Bedenken hinsichtlich der Kosten an.

$\mathrm{Zu}$ den erwarteten wirtschaftlichen Ergebnissen haben die Arbeitsmarktreformen nicht geführt: Es wurde weder ein Wirtschafts- noch ein Beschäftigungswachstum verzeichnet, die Investitionszuflüsse aus dem Ausland nahmen ab. Selbst der IWF räumte in seinem abschließenden Bericht im Juli 2013 ein, dass die Lohnkosten nicht, wie von den Befürwortern der Reformen angenommen, den Zustrom ausländischer Direktinvestitionen hemmten. Dennoch dreht sich das Reformkarussell weiter - im Frühjahr 2014 trat der neu gewählte Premierminister Renzi mit einem neuen Beschäftigungsplan, Jobs Act genannt, an.

\subsection{Der Fall Spanien}

In Spanien setzte die Deregulierung des Arbeitsmarktes kurz nach dem Ende des autoritären Franco-Regimes ein und wurde unter verschiedenen Folgeregierungen, überwiegend sozialistischer Ausrichtung, fortgeführt (Rand Smith 1998). 1984 wurden die Vorschriften für befristete Arbeitsverträge gelockert, woraufhin deren Zahl im Zeitraum 1984 - 1996 von einer halben Million auf über 8 Mio. stieg. 1988, 1992, 1994, 1997 und 2003 kamen weitere Arbeitsmarktreformen hinzu.

Die Auswirkungen der Wirtschaftskrise sind in Spanien besonders deutlich zu spüren. Fiel der Rückgang des BIP 2009 noch leicht geringer aus als in Deutschland oder Italien, so stieg die Arbeitslosigkeit geschwind auf mehr als das Doppelte und erreichte $201225 \%$. Auch das Haushaltsdefizit wuchs rapide und setzte das Land bald dem Druck der Finanzmärkte aus. Während der ersten Griechenlandkrise im Mai 2012 vollzog der sozialistische Premierminister Zapatero eine wirtschafts- und sozialpolitische Kehrtwende und stellte einen Sparhaushalt vor, der zum ersten Mal in der Geschichte des demokratischen Spaniens Lohnund Gehaltskürzungen im öffentlichen Dienst vorsah.

\subsubsection{Intervention der EU}

Dem spanischen Wirtschafts- und Sozialrat angehörende Arbeitgeber- und Gewerkschaftsvertreter äußerten, im Verein mit der EZB habe zu jenem Zeitpunkt auch die Bank von Spanien begonnen, mit großem Nachdruck auf Deregulierung zu drängen (Interviews). Es wurden unter anderem Tarif- und Arbeitsmarktreformen als Zeichen wirtschaftlicher Erholung gefordert. Insbesondere drängte die Bank von Spanien angesichts der Spaltung des Arbeitsmarktes in unbefristete und befristete Arbeitsverhältnisse auf die Einführung eines flexiblen einheitlichen Arbeitsvertrags und rief im Einklang mit EU-Kommissar László Andors ausdrücklicher Empfehlung (El Economista vom 26. April 2011) nach Maßnahmen zur Dezentralisierung der Tarifverhandlungen. Allerdings deutet das Ausmaß des Beschäftigungsrückgangs nicht auf eine mangelnde, sondern eher auf eine zu hohe zahlenmäßige Flexibilität des spanischen Arbeitsmarktes hin. Überdies eröffnet das Gesetz den Unternehmen bereits seit 1994 die Möglichkeit, von Lohnabschlüssen auf Verbandsebene abzuweichen (descuelgue salarial). Und schließlich zeigt sich in den Tarifforderungen spätestens seit 2009 ein Bewusstsein für die Krisensituation (Martín 2010). Der Ruf europäischer Institutionen nach radikaler Deregulierung entspricht zudem nicht der Interessenlage der spanischen Arbeitgeber. Trotz einer gewissen Unterstützung seitens der Escuela Superior de Administración y Dirección de Empresas (ESADE) und anderer Business Schools wird der einheitliche Arbeitsvertrag nicht nur von der Dachorganisation der spanischen Arbeitgeberverbände, Confederación Española de Organizaciones Empresariales (CEOE), abgelehnt, sondern, den Ergebnissen der Unternehmensumfragen Barómetro de Empresas nach zu urteilen, von knapp der Hälfte aller Arbeitgeber. Aus Sicht der Personalleitungen stellt der Festangestelltenstatus angesichts der strukturellen Segmentierung des spanischen Arbeitsmarkts und der spanischen Unternehmensbelegschaften ein wirksames Mittel der Personalsteuerung dar. Diesen Schluss legen zumindest die Erfahrungen mit Mischformen aus fester und flexibler Arbeitszeit nahe, beispielsweise das Scheitern der „Spezialverträge zur Förderung zeitlich unbegrenzter Beschäftigungsverhältnisse" (contratos de fomento de la contratación indefinida), die von den Arbeitnehmern als zweitklassig empfunden werden und sich trotz finanzieller und rechtlicher Anreize nicht durchsetzen konnten. Tariflich gesehen sind die spanischen KMU genauso wenig für Verhandlungen auf Unternehmensebene gerüstet wie der italienische Mittelstand. Folglich sind und bleiben Tarifverträge auf Verbandsebene trotz des Rufs der Arbeitgeber nach mehr Flexibilität unverzichtbar als externer Bezugsrahmen.

\subsubsection{Reaktion der Zapatero-Regierung}

Der spanische Staat reagierte auf die Krise und die im April 2010 übermittelten Vorgaben der EU-Kommission mit einer Reihe von Arbeitsmarktreformen. In einem ersten, im 
Juni 2010 angekündigten einschneidenden Reformschritt wurden die rechtlichen Voraussetzungen für Entlassungen und für Abweichungen von bestehenden Lohnvereinbarungen (descuelgue salarial) sowie die damit verbundenen Kosten erheblich gelockert beziehungsweise reduziert. Die Anreize zum Abschluss von Beschäftigungssicherungsvereinbarungen wurden verstärkt und das in bestimmten Sektoren wie dem Baugewerbe geltende Leiharbeitsverbot wurde aufgehoben. Die Gewerkschaften wehrten sich am 29. September mit einem eher rituell anmutenden Generalstreik. Zum ersten Mal in der spanischen Geschichte hatte ein solcher Ausstand keinerlei Einfluss auf die Beschlüsse der Regierung. Im Februar 2011 wurde eine tripartistische Vereinbarung zum Thema Rentenanpassung getroffen, mit der auch gleichzeitig Verhandlungen über eine Tarifreform angebahnt wurden. Nach einem vielversprechenden Start wurden die Verhandlungen über die Tarifreform jedoch Ende Mai abgebrochen, als der Arbeitgeberverband der Region Madrid, Confederación Empresarial de Madrid (CEIM), als unnachgiebigster unter den Hardlinern des CEOE der erzielten Vereinbarung seine Unterschrift verweigerte. Die Gründe für das Scheitern waren überwiegend politischer Natur: Der Sieg der Rechten bei den Kommunalwahlen am 22. Mai trug ebenso dazu bei wie die Radikalisierung der Linken unter dem Druck der Indignados, einer spontanen Vorläuferbewegung der Occupy-Initiativen.

Nachdem keine Einigung erzielt werden konnte, verabschiedete die Regierung das Reformgesetz einseitig, wenn auch auf Druck der Arbeitgeber und Gewerkschaften hin gewisse Detailänderungen einflossen. In seiner endgültigen Fassung stufte der Gesetzesentwurf Vereinbarungen auf Provinzebene, die im Baugewerbe und anderen nicht landesweit verhandelnden Branchen eine wichtige Rolle spielen, herab und schränkte die automatische Verlängerung auslaufender Tarifverträge ein, womit einer schleichenden Verschlechterung der Beschäftigungsbedingungen Tür und Tor geöffnet wurde. Um sich die ausschlaggebenden Stimmen der autonomistischen Parteien Kataloniens und des Baskenlandes zu sichern, führte die Regierung entgegen den Wünschen der Sozialpartner das Prinzip der Vorrangigkeit regionaler Tarifverträge (convenios autonómicos) ein.

Damit war der Reformprozess jedoch keineswegs abgeschlossen. Wie gesagt, erhöhten sich ab August die Renditeaufschläge für spanische Staatsanleihen, woraufhin die spanische Regierung von der EZB ein Schreiben mit ähnlichem Wortlaut erhielt wie zuvor die italienische. Die Spanier fügten sich und nahmen unverzüglich eine Schuldenbremse in ihre Verfassung auf - ein erstaunlicher Schritt, wenn man bedenkt, dass die spanische Constitución von 1978 mit einer einzigen Ausnahme im Jahre 1992 nie geändert worden war und vielen als unantastbar galt. Am 26. August kündigte die Regierung eine weitere Arbeitsmarktreform an, die den Abschluss befristeter Beschäftigungsverhältnisse erleichtern sollte; sie vollzog damit eine Kehrtwende gegenüber der Reform von 2008 und den „Flexicurity“-Prinzipien, die der Dualisierung des Arbeitsmarktes entgegenwirken sollten.

\subsubsection{Fortsetzung der Reformen unter der konservativen Regierung}

Angesichts sinkender Popularität kündigte die sozialistische Regierung vorgezogene Neuwahlen an, aus denen im November 2011 die konservative Partei Partido Popular als Sieger hervorging. Wie in Italien führte die neue Regierungsmannschaft unverzüglich weitere Austeritätsmaßnahmen ein, auf die im Februar 2012 Arbeitsmarkt- und Tarifreformen folgten. Erst Ende Januar 2012 hatten die Gewerkschaften und Arbeitgeberverbände mit einem neuen landesweiten Lohnabschluss die Anpassungsfähigkeit des spanischen Tarifwesens an den Konjunkturabschwung unter Beweis gestellt, aber der staatliche Reformeifer ließ sich damit nicht bremsen. Die Reform vom Februar 2012 umfasst eine breite Maßnahmenpalette:

- einseitiges Recht der Arbeitgeber auf Schaffung ,interner Flexibilität“" (durch Veränderung von Arbeitsinhalten, Arbeitsort und Arbeitszeiten) ohne vorherige $\mathrm{Zu}$ stimmung der Gewerkschaft(en) oder des Betriebsrates,

- „Arbeitsvertrag zur Unterstützung der Unternehmer“ (contrato de apoyo a los emprendedores), ein neuer unbefristeter Arbeitsvertrag mit einjähriger Probezeit ohne jegliche Beschäftigungssicherheit (ähnlich dem von der französischen Regierung 2005 vorgeschlagenen und später von der Internationalen Arbeitsorganisation verurteilten contrat nouvelles embauches),

- Reduzierung der Abfindungsleistung im Kündigungsfall (einschließlich unbegründeter Kündigung) von 45 auf 20 Tage Gehalt pro Betriebsjahr, Streichung der Überbrückungszahlung für entlassene Arbeitnehmer während der Dauer des arbeitsgerichtlichen Verfahrens sowie Abschaffung der bisher erforderlichen behördlichen Genehmigung von Massenentlassungen,

- absoluter Vorrang betrieblicher Vereinbarungen vor Vereinbarungen auf Verbandsebene sowie Recht der Arbeitgeberseite zur Vornahme von Lohnsenkungen ohne gewerkschaftliche Zustimmung (vorbehaltlich schiedsgerichtlicher Entscheidung),

- Verkürzung der bisher unbegrenzten Nachwirkung (ultraactividad) von Tarifverträgen auf maximal zwei Jahre. Nach Ablauf dieser Frist erlöschen bis zur Unterzeichnung einer neuen Vereinbarung alle Rechte aus früheren Verträgen (in Spanien kamen Verlängerungen durch Nachwirkung um bis zu zehn Jahre vor).

Insgesamt stärkte diese Reform, die im Laufe des Jahres 2012 zwei Generalstreiks auslöste, die Macht der Arbeitgeber in erheblichem Maße und zeitigte 2013 einen Lohnverfall ohne jeglichen Beschäftigungszuwachs. Wie in Italien ist die wirtschaftliche Dringlichkeit der Maßnahmen kaum nachvollziehbar. Die Bilanz der ersten drei Monate ergibt nicht nur die Vernichtung von 179.400 unbefristeten (zuvor weitgehend kündigungsgeschützten) Arbeitsplätzen, sondern entgegen den optimistischen Erwartungen der Reformer überdies einen $10 \%$-igen Rückgang der Zahl neugeschaffener Arbeitsplätze. Arbeitsministerin Fátima Báñez Aufforde- 
rung an die Arbeitgeber, bei der Anwendung der neuen Regelungen „Fingerspitzengefühl“ zu zeigen (El País vom 9. November 2012), kann vor diesem Hintergrund als indirektes Schuldeingeständnis gewertet werden. Zudem hat die Beschneidung administrativer und kollektiver Verfahren zur Regelung von Beschäftigungsbedingungen und Streitbeilegung eine verstärkte Anrufung der Gerichte bewirkt. Die angestrebte Dezentralisierung der Tarifverhandlungen würde das in Anfängen erkennbare Mitbestimmungspotenzial des dualen Arbeitnehmervertretungssystems Spaniens (Vertretung durch Gewerkschaften und Betriebsräte) im Keim ersticken und dafür sorgen, dass integrative Sichtweisen in den Arbeitsbeziehungen auf Unternehmensebene durch Verteilungsdenken verdrängt werden. Im Baugewerbe befürchteten sogar die Arbeitgeber störende Auswirkungen der neuen Regelungen. Sie unterzeichneten deshalb in aller Eile einen neuen Tarifvertrag nach den alten Regeln und sahen sich unversehens in eine juristische Auseinandersetzung mit der Regierung verstrickt, bei der es um die Frage ging, ob der neue Vertrag von der Reform gedeckt sei oder nicht. Im Frühjahr 2013 gestand der Arbeitgeberverband CEOE ein, dass es sinnvoll sei, die Nachwirkung von Tarifverträgen (ultraactividad) zu verlängern, und einigte sich mit den Gewerkschaften auf Maßnahmen zur Vermeidung einer abrupten Ungültigkeit verschiedener Branchentarifverträge.

\section{Schlussfolgerungen}

Durch störende Eingriffe in die bestehenden, wenn auch vielleicht nicht optimalen, Institutionen bewirken die jüngsten Reformen in Ländern Südeuropas eine Verschiebung von lokal eingebetteten Formen des Kapitalismus hin zu weniger in das institutionelle Umfeld eingebundenen Spielarten. Dieser Vorgang birgt ein hohes Risiko unerwünschter Folgen. Es bleibt abzuwarten, ob tatsächlich ein Systemwandel stattfinden wird: Noch ist nicht gesagt, dass die beteiligten Akteure die Reformen auch tatsächlich umsetzen, sei es auf Branchen- oder Firmenebene. In den betroffenen Ländern haben Unstimmigkeiten zwischen den übergeordneten (formellen) und den untergeordneten (informellen) Ebenen eine lange Tradition. Die unteren Ebenen sorgen oftmals für einen Grad an Flexibilität, den die Vorschriften der übergeordneten Instanzen untersagen (Regini 1995). Manche Liberalisierungsbestrebungen der letzten Jahre (in Italien große Teile der italienischen Reformen von 2003 und 2011, in Spanien der contrato de fomento del empleo) wurden nur in sehr geringem Umfang angenommen. Die jüngsten Reformeingriffe zielen jedoch auf das Herzstück des italienischen und spanischen Arbeitsrechtes: koordinierte Tarifverhandlungen und Kündigungsschutz.

Die Entwicklungen in Spanien und Italien sind radikaler verlaufen, als der bislang von diesen Ländern eingeschla- gene Pfad hätte erwarten lassen, und wurden durch äußere Einflüsse, sprich die Europäische Kommission, die EZB und die deutsche Regierung, aktiv gefördert. Wie einer der Hauptfürsprecher der Reformen beklagte (Ichino 2011, S. 98), war in Italien in den späten 2000ern angesichts der Niederlagen der Jahre 2002/2003 gegen den CGIL sowie in Anbetracht der hervorragenden informellen Anpassung an die bestehenden Rechtszwänge weder bei den beiden Parteien des rechten Flügels noch bei den Arbeitgeberverbänden ein Drang zu legislativen Arbeitsmarktreformen zu verspüren. Auch in Spanien hatte der Generalstreik von 2002 seine Ziele erreicht und die konservative Aznar-Regierung zu Änderungen ihres Reformgesetzes gezwungen.

Kurzum, Europäische Kommission und EZB erreichten in wenigen Monaten, was spanische und italienische Arbeitgeber sowie rechtsgerichtete Regierungen nicht einmal zu fordern gewagt hatten. Der Widerstand der Arbeitnehmer, die zuvor gewisse Erfolge errungen hatten, war machtlos im Kampf gegen einen kaum fassbaren, an lokalem politischem Machtgeplänkel uninteressierten und von Generalstreiks unberührten Gegner. Insbesondere das neue Dogma der EZB deckt sich nicht unbedingt mit den Arbeitgeberbedürfnissen in der realen Wirtschaftswelt und verabschiedet sich von der bisherigen Losung „mehr und bessere Arbeitsplätze“ der EU (Europäischer Rat 2000). In der betrieblichen Wirklichkeit sorgt die Kombination von Austeritätsmaßnahmen und Arbeitsmarktderegulierung (wie im Falle der Zeitverträge in Spanien) für „weniger und schlechtere Arbeitsplätze“.

Die Reformen stehen nicht nur im Widerspruch zu den Wünschen der Gewerkschaften und zu etablierten Arbeitnehmerrechten, sondern berühren auch entscheidende Elemente des Produktionssystems vieler spanischer und italienischer Firmen. Sie beschwören auf den jeweiligen Arbeitsmärkten drei Probleme herauf:

(1) Aus Sicht der KMU bedeutet die Dezentralisierung des Tarifwesens eine Erhöhung der Transaktionskosten und behindert durch den resultierenden Lohnwettbewerb die Zusammenarbeit in Industrieclustern. Die durch den Dezentralisierungsprozess bewirkte Verlagerung der Tarifverhandlungen auf die Unternehmensebene wird von den Arbeitgebern der genannten Länder seit Langem abgelehnt - man verhandelt lieber auf Distanz.

(2) Die Lockerung des gesetzlichen Kündigungsschutzes und der Vorrang von Tarifverhandlungen auf Unternehmensebene stellen nicht nur traditionelle paternalistische Formen der Mitarbeiterführung infrage, welche die Belegschaften in treue, mit Anreizen symbolisch-kultureller Art motivierbare Kernmitarbeiter und in flexible Randbeschäftigte spalten. Durch die Reformen wird die Gefahr einer Schwächung der Loyalität von Kernmitarbeitern heraufbeschworen, gekoppelt mit einer potenziell geringeren Macht über befristet Beschäftigte. Sollte daraus die Notwendigkeit erwachsen, Mitarbeiter verstärkt mit monetären Anreizen zu motivieren, wäre ein Kostenanstieg unvermeidlich. 
(3) Die neuen Bestimmungen untergraben Vertrauensverhältnisse und informelle Ressourcen, die für lokale, auf ein stabiles und kalkulierbares Umfeld angewiesene Produktionssysteme wie Industriecluster eine entscheidende Rolle spielen.

Insgesamt zeichnet sich ein Reformmuster ab, das aus Sicht einiger weniger, schwach in das lokale Umfeld eingebundener Unternehmen seine Vorteile haben mag, viele andere Arbeitgeber aber ihrer Grundlagen beraubt. Die durch äußeren Druck bedingte Überstürzung, mit der diese Reformen durchgepeitscht wurden, macht deren Umsetzung schwierig: Trotz der damit verbundenen Liberalisierung schleppt sich die viel beschworene Dezentralisierung der Tarifverhandlungen auf bleiernen Füßen voran. Ob sich die tarifliche Deckungsrate wesentlich verändern wird, lässt sich noch nicht sagen. Zwar schwächen die Reformeingriffe traditionelle Formen des Widerstands und der Kompromissfindung, längerfristig jedoch werden sie voraussichtlich zu größerer Konfliktbereitschaft und höherer Verunsicherung führen. In beiden Ländern zeichnet sich eine verstärkte Fragilität der Branchentarifverträge ab: 2013 wurde in mehreren Fällen die Erneuerung bestehender Vereinbarungen hinausgeschoben, die Arbeitgeber des italienischen Bankensektors kündigten ihren Vertrag einseitig auf und in Spanien stieg die Anzahl vom Tarifvertrag abweichender Lohnabschlüsse um das Siebenfache (Quelle: Ministerio de Empleo).

Neben vergleichbaren Entwicklungen in Italien und Spanien gibt es jedoch auch Unterschiede zwischen diesen Ländern, die nicht dem Klischee eines einheitlichen mediterranen Modells entsprechen: In Italien sind noch immer die Verbände die treibende Kraft, in Spanien dagegen Staat und Regierungsmacht. Dies erklärt sowohl den schnelleren Anstieg der Arbeitslosigkeit als auch die größere Tiefe und Einseitigkeit der Reformen im Vergleich zu Italien. Die Unterschiede zeigen, dass äußere Einflüsse ungeachtet ihrer gestiegenen Bedeutung die vorhandenen Institutionen und Traditionen eines Landes nicht einfach aushebeln können und auch keine simple neoliberale Konvergenz nach sich ziehen.

Mit Blick auf die Zukunft sind zwei alternative Szenarien denkbar, die den beschriebenen Wandlungsprozess aufhalten könnten. Die eine Möglichkeit wäre, dass sich auf lokaler Ebene institutioneller Widerstand formiert - weniger gegen die Reformen an sich als gegen deren Umsetzung. Vereinte Bemühungen von Gewerkschaften, Arbeitgeberblöcken, Kommunalbehörden und Kirche sowie eventuell ein gewisses Maß wohlwollender Nachlässigkeit seitens der Regierungsbehörden könnten die Reformen im Alltag bremsen und dafür sorgen, dass die betriebliche Praxis nicht allzu sehr von den Änderungen auf dem Papier gestört wird. Im zweiten denkbaren Szenario würde radikaler Widerstand aufkeimen, entweder bei den Gewerkschaften oder in Gestalt von neuen sozialen Bewegungen wie den Indignados, in populistischen Parteien oder auch in Form von unorganisierten oppositionellen Praktiken und Widerständen der Arbeitnehmer wie z. B. nachlassendes Engagement und erhöhte Bereitschaft zum Arbeitsplatzwechsel. Im Herbst 2012 erzwangen Massenproteste in Portugal und Spanien gewisse Regierungszugeständnisse, in ganz Südeuropa schwindet rapide das Vertrauen in die etablierten politischen Parteien. Die Wahrscheinlichkeit, dass der Widerstand gegen die Reformen Erfolg hat, ist angesichts der bestehenden Unterschiede allerdings in Italien höher als in Spanien.

\section{LITERATUR}

Amable, B. (2003): The diversity of modern capitalism, Oxford Berton, F./Richiardi, M./Sacchi, S. (2012): The political economy of work security and flexibility, Bristol

Crouch, C. (1993): Industrial relations and European state traditions, Oxford Crouch, C. (2011): The strange non-death of neoliberalism, Oxford

Erne, R. (2012): European industrial relations after the crisis. A postscript, in Sismans, S. (Hrsg.): The European Union and industrial relations - new procedures, new context, Manchester, S. 225-235

Esping-Andersen, G./Regini, M. (Hrsg.) (2000): Why deregulate labour markets?, Oxford

Europäische Kommission (2006): Employment in Europe 2006, Luxembourg Office for Official Publications of the European Communities, Strasbourg Europäische Kommission (2010): An agenda for new skills and jobs, Strasbourg Europäische Kommission (2012): Wage setting systems and wage developments, Brussels, http://ec.europa.eu/europe2020/pdf/themes/wage_settings.pdf Europäischer Rat (2000): Presidency conclusions: Lisbon European Council, 23. and 24. March

Hall, P. A./Soskice, D. (Hrsg.) (2001): Varieties of capitalism: the institutional foundation of comparative advantage, Oxford

Ichino, P. (2011): Inchiesta sul lavoro, Milan

Klein, N. (2008): The shock doctrine, New York

Martín, R. B. (2010): La incidencia de la crisis económica en la negociación coletiva, in: Martín, R. B. (Hrsg.): La negociación colectiva ante la crisis económica, Albacete, S. 9-34

Neguerla, E. (2012): Un nuevo papel en Europa para los países del sur, Informes de la Fondación (47), Madrid

Rand Smith, W. (1998): The left's dirty job: the politics of industrial restructuring in France and Spain, Toronto

Regini, M. (1995): Uncertain boundaries: the social and political construction of European economies, Cambridge

Simonazzi, A. (2012): Italy: chronicle of a crisis foretold', in: Lehndorff, S. (Hrsg.) A triumph of failed ideas. European models of capitalism in the crisis, Brussels, S. $183-198$

Stiglitz, J. (2002): Globalization and its discontents, New York

Streeck, W. (1995): Neo-voluntarism: a new European policy regime, in: European Law Journal 1 (1), S. 31-59

Streeck, W. (2009): Re-forming capitalism: Institutional change in the German political economy, Oxford

Streeck, W./Thelen, K. (Hrsg.) (2005): Introduction: institutional change in advanced political economies, in: Streeck, W./Thelen, K. (Hrsg.): Beyond continuity: institutional change in advanced political economies, Oxford, S. 3-39 Upchurch, P./Weltman, D. (2008): International financial institutions and post communist labour reform: A case of utopian liberalism?, in: Debatte 16 (3), S. 309-330 Thompson, P. (2003): Disconnected capitalism: or why employers can't keep their side of the bargain, in: Work, Employment and Society 17 (2), S. 359-378 Traxler, F./Blaschke, S./Kittel, B. (2001): National labour relations in international markets, Oxford

Trichet, J.-C./Draghi, M. (2011): Un'azione pressante per ristabilire la fiducia degli investitori, in: Corriere della Sera, 29th September, http://www.corriere.it/economia/11_settembre_29/trichet_draghi_inglese_ 304a5f1e-ea59-11e0-ae06-4da866778017.shtml?fr=correlati

\section{AUTOR}

GUGLIELMO MEARDI, Professor für Industrial Relations und Direktor der Forschungseinheit Industrial Relations an der Warwick Business School. Arbeitsschwerpunkte: Internationalisierung der Arbeitsbeziehungen - mit Bezug zu internationalen Institutionen, multinationalen Unternehmen und Arbeitsmigration.

guglielmo.meardi@wbs.ac.uk 